\title{
CONFIRMAÇÃO DA IDENTIDADE DA $\alpha$-CRIPTOXANTINA E INCIDÊNCIA DE CAROTENÓIDES MINORITÁRIOS PROVITAMÍNICOS A EM VERDURAS FOLHOSAS VERDES ${ }^{1}$
}

\author{
Adriana Z. MERCADANTE2*, Délia RODRIGUEZ-AMAYA²
}

\begin{abstract}
RESUMO
Numerosos trabalhos comprovaram que os carotenóides principais de folhas verdes são invariavelmente luteína, $\beta$-caroteno, violaxantina e neoxantina. No entanto, há discordância em torno dos carotenóides minoritários. Portanto, a espectrometria de massas por impacto de elétrons e cromatografia líquida de alta eficiência com detector de arranjo de diodos foram utilizados para confirmar a identidade de carotenóides minoritários com atividade provitamínica A em verduras folhosas brasileiras. Os carotenóides pró-vitamínicos $\mathrm{A}$, incluindo os isômeros cis e trans de $\beta$-caroteno, foram separados em coluna de $C_{18}$ polimérica, Vydac 201TP54, com metanol/ água (98:2) como fase móvel. Os espectros UV-visível e de massas confirmaram o carotenóide monoidroxilado como sendo $\alpha$-criptoxantina e não $\beta$-criptoxantina como aponta a literatura internacional. Todas as onze folhas analisadas (agrião, alface crespa, alface lisa, almeirão, caruru, chicória, couve, espinafre, rúcula, salsinha e taioba) apresentaram $\alpha$-criptoxantina, 13-cis$\beta$-caroteno e 9 -cis- $\beta$-caroteno, enquanto que $\alpha$-caroteno foi encontrado em apenas quatro folhas (caruru, couve, salsinha e taioba).
\end{abstract}

Palavras-chave: carotenóides; espectrometria de massas; cromatografia líquida de alta eficiência; verduras folhosas verdes.

\section{SUMMARY}

CONFIRMATION OF THE IDENTITY OF $\alpha$-CRYPTOXANTHIN AND INCIDENCE OF MINOR PROVITAMIN A CAROTENOIDS IN GREEN LEAFY VEGETABLES. The main carotenoids from green leafy vegetables have been consistently found to be lutein, $\beta$-carotene, violaxanthin and neoxanthin. However, there is a controversy about the identity of minor carotenoids. Therefore, electron impact mass spectrometry and high performance liquid chromatography with a diode array detector were used in order to confirm the identity of the minor provitamin A carotenoids in Brazilian green leaves. The provitamin A carotenoids, including the cis and trans isomers of $\beta$-carotene, were separated on a polymeric $\mathrm{C}_{18}$ column, Vydac 201TP54, with $\mathrm{MeOH} / \mathrm{H}_{2} \mathrm{O}$ (98:2) as mobile phase. The UV-visible and mass spectra confirmed that the monohydroxy carotenoid present in Brazilian green leafy vegetable to be $\alpha$-cryptoxanthin, and not $\beta$-cryptoxanthin as reported in the international literature. All eleven green leaves analyzed (water-cress, unheaded lettuce, lettuce, wild chicory, "caruru", common chicory, kale, spinach, endive, roquette, parsley, and "taioba") had $\alpha$-cryptoxanthin, 9 -cis and 13 -cis- $\beta$-carotene, whereas $\alpha$-carotene was found in only four of these leaves ("caruru", kale, salsinha and "taioba").

Keywords: carotenoids; mass spectrometry; high performance liquid chromatography; green leafy vegetables.

\footnotetext{
${ }^{1}$ Recebido para publicação em 25/01/00. Aceito para publicação em 18/05/01.Parte da Tese de Doutorado da $1^{\text {a }}$ autora, realizada junto ao Curso de Pós-Graduação em Ciência de Alimentos da FEA/UNICAMP

2 Professora do Departamento de Ciência de Alimentos, Faculdade de Engenharia de Alimentos, UNICAMP, Caixa Postal 6121, Campinas, 13083-970. E-mail:mercadan@obelix.unicamp.br ${ }^{*}$ A quem a correspondência deve ser enviada.
}

\section{1 - INTRODUÇÃO}

Segundo GOODWIN [18], os tecidos de plantas verdes superiores possuem quatro carotenóides principais: $\beta$-caroteno, luteína, violaxantina e neoxantina. Esta afirmação foi comprovada em numerosos trabalhos. No entanto, há discordância, em torno dos carotenóides minoritários. Os pigmentos que são esporadicamente relatados, em pequenas quantidades, são: cis-isômeros de $\beta$-caroteno, $\alpha$-caroteno, $\beta$-criptoxantina, zeaxantina, anteraxantina e 5,6-epóxi-luteína.

ROUCHAUD et al [36] e o grupo de RODRIGUEZAMAYA [24, 25, 34] foram os únicos pesquisadores que encontraram $\alpha$-criptoxantina ao invés de $\beta$-criptoxantina em folhas verdes. Nos trabalhos citados acima, a identificação foi baseada no espectro de absorção na região visível $\left(\lambda_{\max }\right.$ e forma), nas propriedades cromatográficas e nos três últimos estudos [24, 25, 34], também na reação de metilação que indicou a posição alílica da hidroxila. Como existem três possiveis carotenóides monoidroxilados (Figura 1), ainda existe na literatura internacional uma confusão sobre a identidade do carotenóide monoidroxilado presente em folhas verdes, sendo que na maioria dos trabalhos foi escolhido um ou outro sem a devida comprovação.<smiles>CC1=C(/C=C/C(C)=C/C=C/C(C)=C/C=C/C=C(C)/C=C/C=C(C)/C=C/C2=C(C)CC(O)CC2(C)C)C(C)(C)CCC1</smiles>

$\beta$-criptoxantina<smiles>CC1=CC(O)CC(C)(C)C1/C=C/C(C)=C/C=C/C(C)=C/C=C/C=C(C)/C=C/C=C(C)/C=C/C1=C(C)CCCC1(C)C</smiles>

$\alpha$-criptoxantina<smiles>CC1=CCCC(C)(C)C1/C=C/C(C)=C/C=C/C(C)=C/C=C/C=C(C)/C=C/C=C(C)/C=C/C1=C(C)CC(O)CC1(C)C</smiles>

zeinoxantina

FIGURA 1. Estrutura dos carotenóides monoidroxilados: $\beta$-criptoxantina, $\alpha$-criptoxantina e zeinoxantina.

A introdução da cromatografia líquida de alta eficiência (CLAE) na área de carotenóides em alimentos 
permitiu a separação de um maior número de compostos, incluindo diversos isômeros geométricos, tornando ainda mais difícil a identificação conclusiva destes compostos. Desta forma, um grande número de trabalhos na literatura tem apresentado identificações equivocadas.

Um conjunto de parâmetros é utilizado para a identificação dos carotenóides: (a) espectro de absorção no UV-visível, (b) ordem de eluição na coluna cromatográfica, (c) co-cromatografia com amostras autênticas e (d) espectro de massas [23]. O uso do detector de arranjo de diodos (DAD) permite a obtenção "on line" dos espectros de absorção. A espectrometria de massas (EM) é uma técnica altamente recomendada para a confirmação da identidade de carotenóides e para a elucidação da estrutura de carotenóides desconhecidos, pois quase sempre fornece a massa molecular e alguns dos fragmentos obtidos por impacto de elétrons são indicativos dos grupos terminais. A EM foi largamente utilizada para a elucidação ou confirmação das estruturas de carotenóides em fungos, algas e bactérias. O seu maior uso para carotenóides em alimentos é um acontecimento mais recente [26, 27, 28, 29, 30, 31, 32].

No presente trabalho, os parâmetros mencionados acima foram utilizados para confirmar a identidade dos carotenóides minoritários provitamínicos $\mathrm{A}$ em folhas verdes.

\section{2 - MATERIAL E MÉTODOS}

\section{1 - Amostras}

As seguintes verduras folhosas foram compradas no comércio de Campinas e imediatamente levadas ao laboratório para análise: alfaces crespa e lisa (Lactuca sativa), caruru (Amaranthus viridis), couve (Brassica oleracea var. acephala), taioba (Xanthosoma spp.), agrião (Nastrutium officinale), salsinha (Petroselium hortense), rúcula (Eruca sativa), almeirão (Chicorium intybus), chicória (Chicorium endivia) e espinafre (Spinacea oleracea). Cada maço de folha foi triturado separadamente em multiprocessador e retirada a quantidade necessária de amostra para análise por CLAE (aproximadamente $5 \mathrm{~g}$ ).

A $\alpha$-criptoxantina foi extraída de dois maços de caruru (aproximadamente $100 \mathrm{~g}$ ), pois esta folha foi a que apresentou o maior teor de $\alpha$-criptoxantina dentre as várias folhas previamente analisadas no Brasil [24, 25, 34].

\section{2 - Etapas pré-cromatográficas}

As etapas de extração e saponificação foram executadas de acordo com o método de RODRIGUEZAMAYA et al [35]. Apesar da saponificação não ter sido necessária para a separação dos carotenóides de foIhas [24, 25], esta etapa foi incluída no presente trabaIho com o intuito de eliminar lipídeos e clorofila a fim de facilitar o posterior isolamento e visualização dos carotenóides minoritários.

\section{3 - Isolamento da $\alpha$-criptoxantina}

Para prevenir a contaminação dos carotenóides desde a extração até o isolamento final, não foram usadas garrafas e pissetas plásticas, e a água destilada foi recolhida em recipiente de vidro [3,6]. Além disso, todos os solventes grau P.A. foram destilados, e os peróxidos removidos do éter etílico (EE) pela adição de ferro reduzido antes da destilação. O EE, assim purificado, foi utilizado dentro de cinco dias.

Após extração dos carotenóides de caruru, o extrato saponificado foi aplicado em uma coluna de vidro, empacotada à vácuo com $\mathrm{MgO} /$ Hyflosupercel (1:2) até a altura de cerca de $10 \mathrm{~cm}$. Uma pequena quantidade de sulfato de sódio anidro foi adicionada ao topo da coluna para reter a água que ainda estivesse presente na amostra. A coluna foi molhada com éter de petróleo (EP) e, após aplicação da amostra, desenvolvida com proporções crescentes de EE em EP. A banda contendo $\alpha$-criptoxantina foi eluída com 10 a $30 \%$ de EE em EP.

Após evaporação do solvente, a fração contendo $\alpha$-criptoxantina foi acondicionada em ampola âmbar, selada sob atmosfera de $\mathrm{N}_{2}$ e transportada, sem refrigeração, à Inglaterra. O carotenóide foi purificado por cromatografia em camada delgada de sílica, com EP:EE (3:2) como fase móvel. A banda principal, apresentando cor amarela e $R_{f}, 0,7$, foi raspada e o carotenóide rapidamente extraído com $E E$, em funil de vidro sinterizado sob vácuo.

Imediatamente antes da EM, a $\alpha$-criptoxantina foi re-purificada através de uma mini-coluna de alumina (grau III). Utilizou-se como fase móvel 10\% de EE em EP para retirada de possíveis compostos menos polares que ainda estivessem presentes, e a $\alpha$-criptoxantina foi eluída com 25 a $50 \%$ de EE em EP.

\section{4 - Espectrometria de massas}

Utilizou-se um espectrômetro de massas marca VG (VG Biotech., UK) modelo Quattro Triple Quadrupole. O carotenóide foi introduzido diretamente por sonda na câmara de ionização a $240^{\circ} \mathrm{C}$. Empregou-se ionização por impacto de elétrons, com energia de $70 \mathrm{eV}$.

A interpretação do espectro de massas foi baseada nas fragmentações características, disponíveis na literatura $[14,15,16]$.

\section{5 - Cromatografia líquida de alta eficiência}

Utilizou-se um cromatógrafo líquido Varian, com sistema ternário de bombeamento de solvente, modelo 9010 , e válvula injetora "Rheodyne" com alça de amostra de $10 \mu \mathrm{L}$ de capacidade. O DAD da Waters, modelo 994, foi acoplado a um integrador-registrador Varian, modelo 4400. Os carotenóides foram separados em coluna polimérica

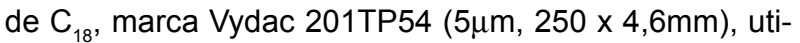
lizando como fase móvel metanol/água (98:2) com vazão de $1,5 \mathrm{~mL} / \mathrm{min}$ [17]. Os cromatogramas foram obtidos a $445 \mathrm{~nm}$ e os espectros entre 300 e $600 \mathrm{~nm}$.

Os comprimentos de onda de absorção máxima $\left(\lambda_{\max }\right)$ e grau de estrutura fina (\%III/II) dos espectros na região do visível foram comparados com os da literatura $[5,13]$ fornecendo uma primeira indicação da identidade dos carotenóides. 
Para co-cromatografia foram utilizados padrões de $\beta$-caroteno e $\beta$-criptoxantina (Hoffmann-La Roche), e $\alpha$-caroteno isolado de cenoura.

Todos os solventes utilizados, de grau cromatográfico, foram previamente filtrados à vácuo, com membrana para solvente orgânico de $0,45 \mu \mathrm{m}$ e, a seguir, desgaseificados em ultra-som. A água utilizada foi obtida pelo sistema de purificação e filtração Milli-Q (Millipore). Todas as amostras foram previamente filtradas em membrana de $0,45 \mu \mathrm{m}$.

\section{3 - RESULTADOS E DISCUSSÃO}

3.1 - Confirmação da identidade e incidência de $\alpha$ criptoxantina

O espectro de absorção no visível, fornecido pelo DAD, da $\alpha$-criptoxantina apresentou $\lambda_{\max }(420,445$,

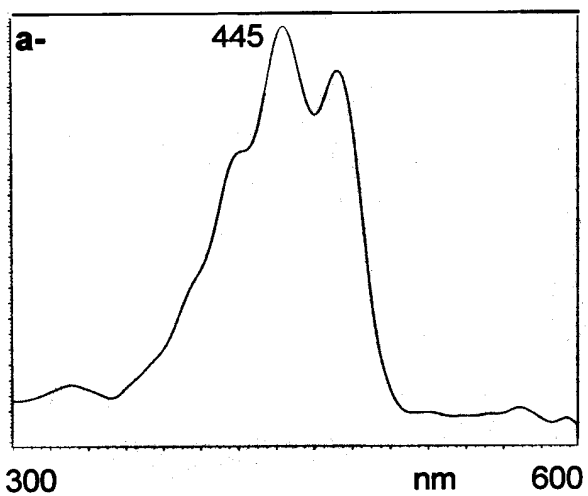

$472 \mathrm{~nm})$ e estrutura fina similares aos do $\alpha$-caroteno (Figura 2), fornecendo uma primeira identificação.

$\mathrm{O}$ espectro de massas da $\alpha$-criptoxantina (Figura 3) mostrou o íon molecular a m/z $552(0,4 \%)$ e o fragmento característico de eliminação de água a $\mathrm{m} / \mathrm{z} 534$ $(0,7 \%)$, indicando a presença de uma hidroxila na molécula. A maior intensidade do pico de 534 unidades de massa em relação ao do íon molecular ([M] ${ }^{+} /[\mathrm{M}-18]^{+}$ de 0,59 ) indicou que a hidroxila se encontrava em posição alílica. BRITTON \& YOUNG [6] também reportaram valor abaixo de 1,0 para a razão citada acima para $\alpha$-criptoxantina isolada de tecidos fotossintéticos. Devido à baixa concentração deste carotenóide, os picos de eliminação de tolueno e xileno da cadeia poliênica não foram observados (Figura 3).

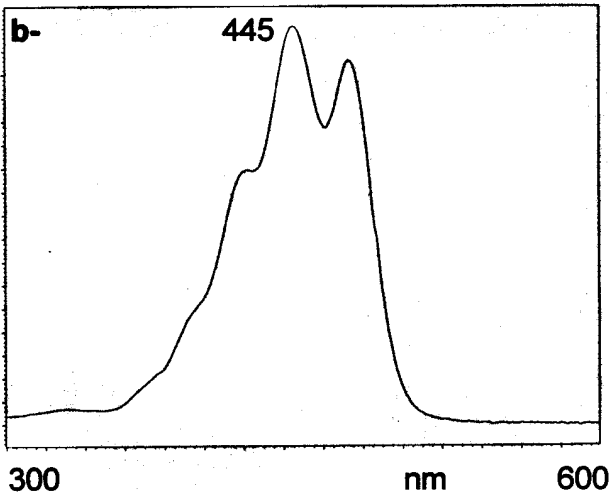

FIGURA 2. Espectros de absorção, obtidos pelo DAD, em metanol-água (98:2), da a- $\alpha$-criptoxantina e do b- $\alpha$-caroteno.

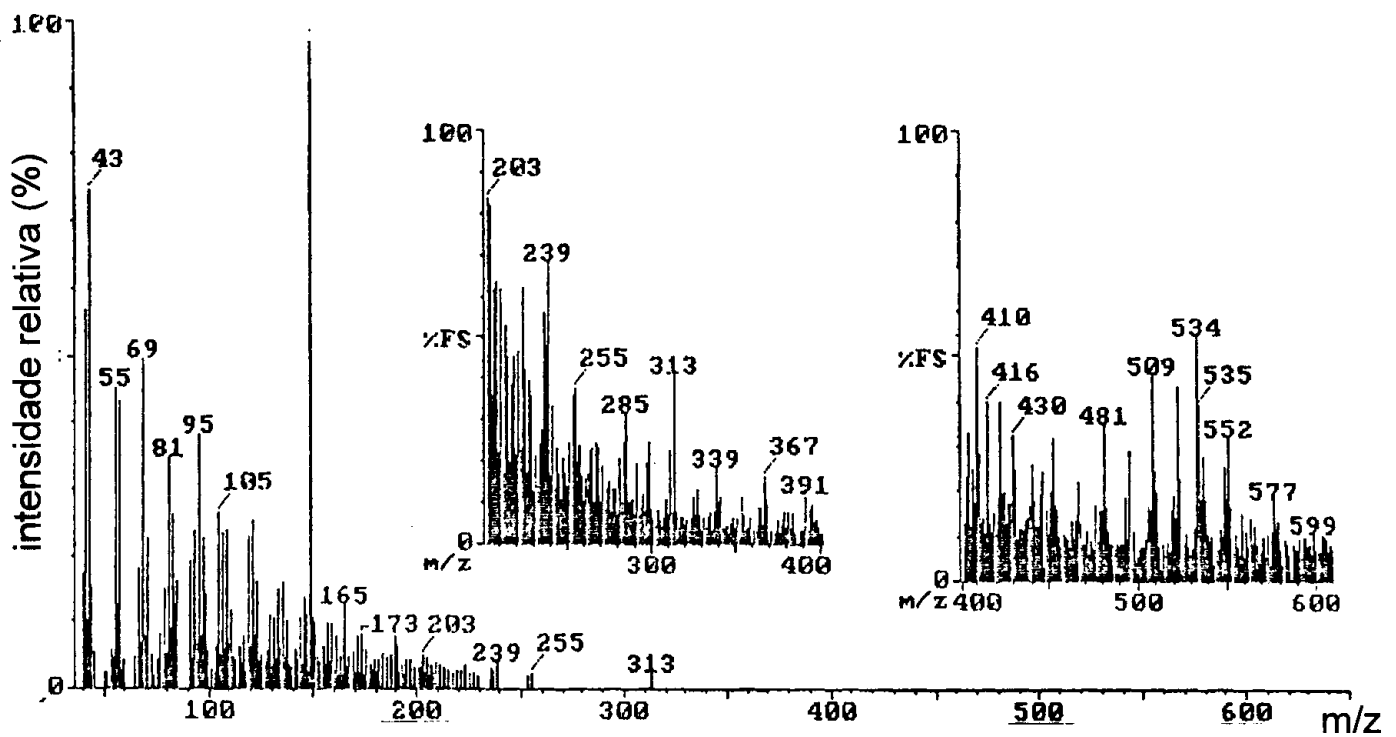

FIGURA 3. Espectro de massas de impacto de elétrons $(70 \mathrm{eV})$ da $\alpha$-criptoxantina, isolada de caruru.

Todas as verduras folhosas analisadas neste estudo apresentaram $\alpha$-criptoxantina ao invés de $\beta$ criptoxantina (Figura 4).

Esta é a primeira comprovação definitiva da presença de $\alpha$-criptoxantina em plantas superiores. Até então, acreditava-se que o derivado monoidroxilado do $\alpha$-caroteno era zeinoxantina em plantas superiores, e $\alpha$-criptoxantina em alga vermelha [20].

\section{2 - Incidência de $\alpha$-caroteno}

Das 11 verduras folhosas verdes analisadas neste estudo, $\alpha$-caroteno foi encontrado somente em 4 delas 
(caruru, couve, salsinha e taioba), como exemplificado na Figura 4.

Além do espectro obtido pelo DAD (Figura 2), foi realizada co-cromatografia com $\alpha$-caroteno isolado de cenoura (Figura 5).

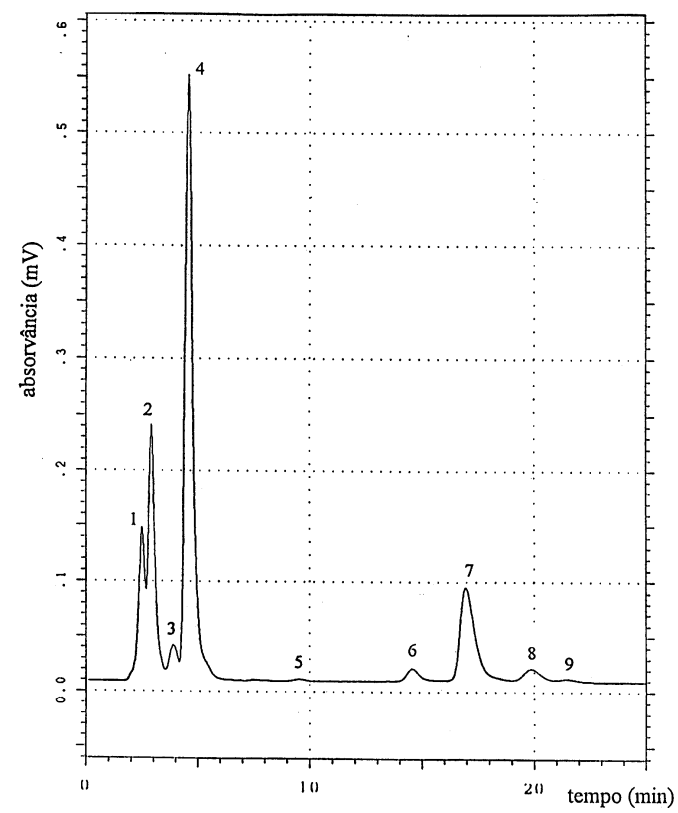

FIGURA 4. Cromatograma, obtido por CLAE, dos carotenóides de taioba. Picos: 1, 2, 3 e 4: poliidroxilados, 5: $\alpha$-criptoxantina, 6: $\alpha$-caroteno, 7 : $\beta$-caroteno, 8: 13-cis- $\beta$-caroteno e 9: 9cis- $\beta$-caroteno. Condições cromatográficas: coluna Vydac

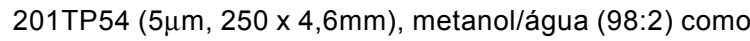
fase móvel com vazão de $1,5 \mathrm{~mL} / \mathrm{min}$.

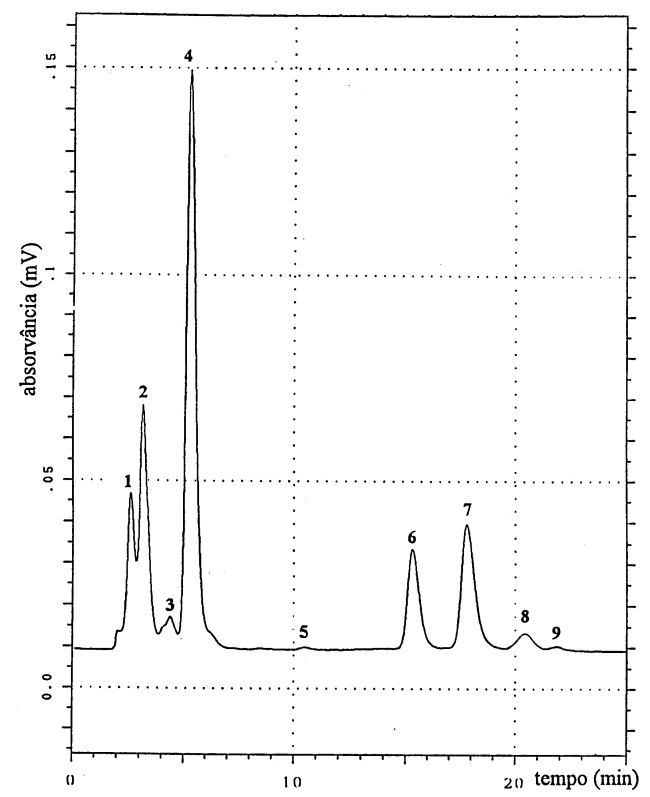

FIGURA 5. Cromatograma, obtido por CLAE, do extrato de taioba fortificado com $\alpha$-caroteno. Picos: 1, 2, 3 e 4: poliidroxilados, 5: $\alpha$-criptoxantina, 6 : $\alpha$-caroteno, $7: \beta$-caroteno, 8 : 13 -cis- $\beta$ caroteno e 9: 9-cis- $\beta$-caroteno. Condições cromatográficas: coluna Vydac 201TP54 ( $5 \mu \mathrm{m}, 250$ × 4,6mm), metanol/água (98:2) como fase móvel com vazão de $1,5 \mathrm{~mL} / \mathrm{min}$.
Dos inúmeros estudos encontrados na literatura sobre composição de carotenóides em folhas, verifica-se que, para a mesma folha, há diferentes relatos quanto à presença de $\alpha$-caroteno, mesmo entre aqueles que empregaram o mesmo método de separação. A Tabela 1 apresenta esses trabalhos para quatro folhas, nos quais utilizou-se CLAE para a separação dos carotenóides. Dos sete estudos com espinafre, $\alpha$-caroteno foi encontrado, em traços, apenas em um deles. Em couve e salsinha não foi detectado $\alpha$-caroteno. Entretanto, em alface, a situação é dividida, já que três dos seis estudos apresentaram $\alpha$-caroteno com teores variando de traços a $5,15 \mu \mathrm{g} / 100 \mathrm{~g}$ de amostra.

Como o $\alpha$-caroteno esteve presente em pequenas quantidades, quando detectado, esta disparidade nos resultados pode ser explicada pelos diferentes níveis de detecção dos métodos e/ou pelo comprovado efeito das diferentes espécies e cultivares, do clima e do solo sobre o teor de carotenóides e presença dos minoritários em folhas [24]. Os resultados obtidos no presente trabalho indicam ser a segunda explicação a mais provável, uma vez que as condições analíticas empregadas foram as mesmas para as 11 folhas estudadas.

Por outro lado, ALMEIDA-MURADIAN et al [2] relataram a presença de altos teores de $\alpha$-caroteno em foIhas de cenoura, chegando a $29 \%$ do total de carotenos em uma das cultivares. Esta alta porcentagem de $\alpha$ caroteno, nunca relatada anteriormente por outros autores, aproxima-se mais da porcentagem deste carotenóide na raiz. Os autores separaram $\alpha$-e $\beta$-caroteno por CLAE em fase reversa, mas a quantificação destes dois carotenos foi executada após separação em coluna de MgO:hyflosupercel.

TABELA 1. Incidência de $\alpha$-caroteno em 4 verduras folhosas reportada da literatura.

\begin{tabular}{ccc}
\hline Folha & $\alpha$-caroteno & Referência $^{\text {a }}$ \\
\hline alface & $x$ & BUREAU \& BUSHWAY [7] \\
& - & BUSHWAY [9] \\
& $x$ & HEINONEN et al [21] \\
& $x$ & ABDEL-KADER [1] \\
& - & TEE \& LIM [37] \\
& - & GRANADO et al [19] \\
\hline espinafre & - & presente trabalho \\
& - & BRAUMANN \& GRIMME [4] \\
& - & BUREAU \& BUSHWAY [7] \\
& - & KHACHWH et al [22] \\
& - & HEINONEN et al [21] \\
& - & ABDEL-KADER [1] \\
& - & TEE \& LIM [37] \\
& - & GRANADO et al [19] \\
& - & NYAMBAKA \& RYLEY [33] \\
& - & presente trabalho \\
couve & $x$ & BUSHWAY [8] \\
& - & KHACHIK et al [22] \\
& - & CARVALHO et al [10] \\
& $x$ & presente trabalho \\
\hline salsinha & - & HEINONEN et al [21] \\
& - & YAMAUCHI \& WATADA [39] \\
& $x$ & presente trabalho \\
& &
\end{tabular}

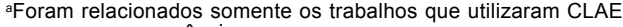
$\mathrm{x}$ : presença; -: ausência. 


\section{3 - Incidência de cis isômeros de $\beta$-caroteno}

Os carotenóides geralmente ocorrem na natureza na sua forma mais estável, que é a configuração trans. Os cis isômeros também podem existir naturalmente, em pequenas quantidades, e como possuem menor biopotência, a sua quantificação em separado vem sendo discutida.

No presente trabalho foi utilizado uma coluna $\mathrm{C}_{18}$ polimérica (Vydac 201TP54) capaz de separar os isômeros cis de $\beta$-caroteno [8]. A identificação foi baseada nos espectros de absorção fornecidos pelo DAD (Figura 6), pois os isômeros cis apresentam $\lambda_{\text {max }}$ ligeiramente mais baixos e decréscimo no grau de estrutura fina do que a forma trans, aparecimento do pico cis na região ultravioleta e comparação da ordem de eluição na coluna com os trabalhos de BUSHWAY [8] e de GODOY \& RODRIGUEZ-AMAYA [17] que utilizaram exa- tamente as mesmas condições cromatográficas empregadas neste estudo. Neste último trabalho [17] a identidade dos isômeros cis foi confirmada por cocromatografia com padrões isolados por cromatografia em coluna aberta de hidróxido de cálcio, pois já havia sido demonstrado através de ressonância magnética nuclear de próton e carbono 13 a identidade dos isômeros de $\beta$-caroteno separados neste mesmo tipo de coluna [38].

Todas as verduras folhosas verdes analisadas no presente estudo apresentaram 9-cis-e 13-cis- $\beta$-caroteno (Figura 4), confirmando os registros da literatura [11, 12, 17, 33]. KHACHIK et al [22] relataram a presença de 15 -cis- $\beta$-caroteno em quatro vegetais verdes, entretanto, estes mesmos autores reconheceram, mais tarde, que se tratava na verdade de uma mistura de 13-cise 15-cis- $\beta$-caroteno.
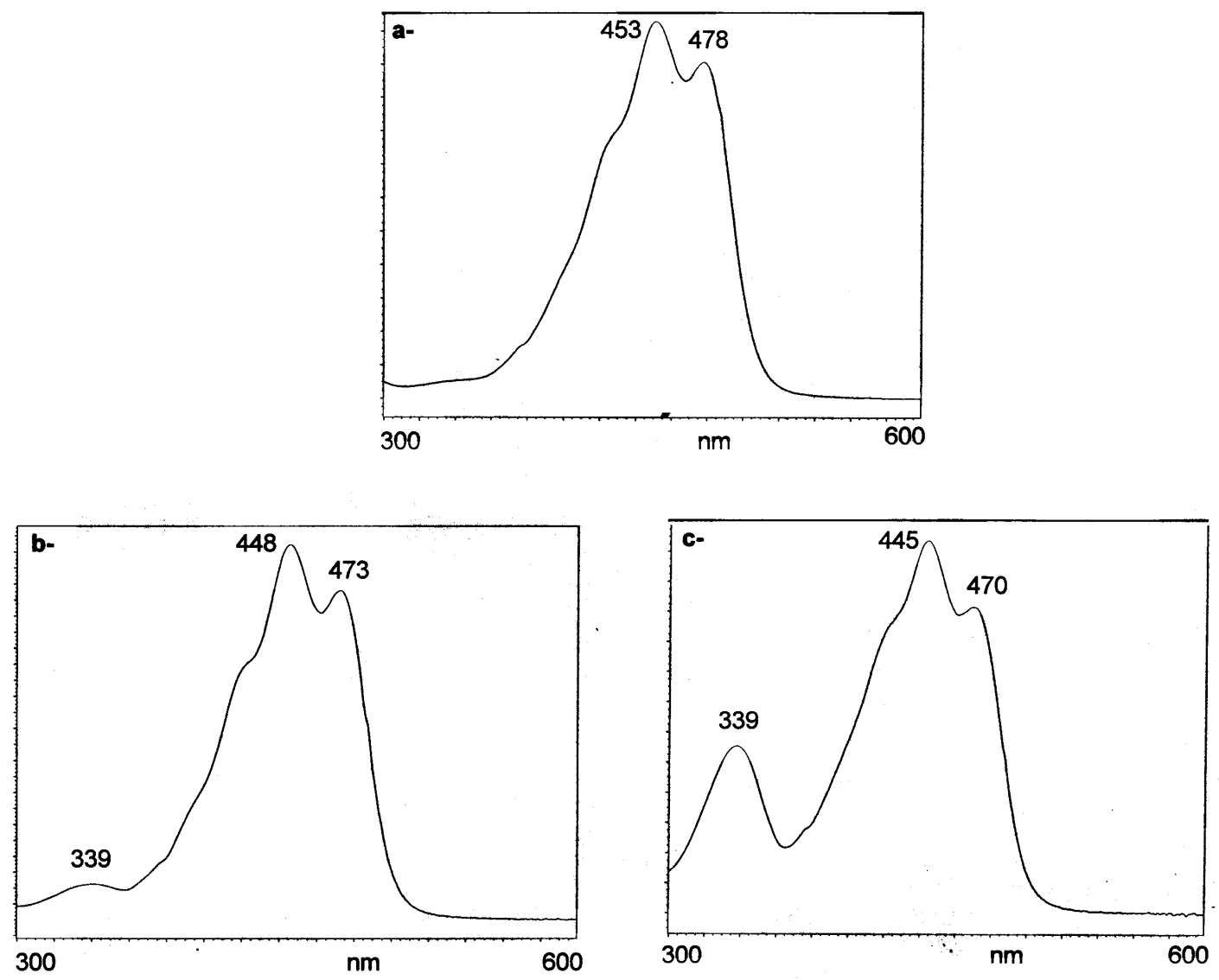

FIGURA 6. Espectros de absorção, obtidos pelo DAD, em metanol-água (98:2), da a- $\alpha$ - all-trans- $\beta$-caroteno, b- 9 -cis- $\beta$-caroteno e c13-cis- $\beta$-caroteno.

\section{4 - CONCLUSÕES}

- A identidade do carotenóide monoidroxilado das verduras folhosas verdes foi confirmada inequivocadamente como sendo $\alpha$-criptoxantina.
- Todas as onze verduras folhosas verdes analisadas apresentaram os seguintes carotenóides minoritários próvitamínicos A: $\alpha$-criptoxantina, 9-cise 13-cis- $\beta$-caroteno.

- Somente caruru, couve, salsinha e taioba apresentaram $\alpha$-caroteno. 


\section{5 - REFERÊNCIAS BIBLIOGRÁFICAS}

[1] ABDEL-KADER, Z.M. Determination of carotenoids in foods by high-performance liquid chromatography. Die Nahrung, v.35, p.689-93, 1991.

[2] ALMEIDA-MURADIAN, L.B.; POPP, V. \& FARIAS, M.P. Provitamin A activity of Brazilian carrots: leaves and roots, raw and cooked and their chemical composition. Ciênc. Tecnol. Aliment., v.17, p. 120-4, 1997.

[3] BIEMANN, K. Mass Spectrometry. In: Organic Chemical Applications. New York: Mc Graw Hill, 1962. p. 170-173.

[4] BRAUMANN, T. \& GRIMME, L.H. Reversed-phase highperformance liquid chromatography of chlorophylls and carotenoids. Biochim. Biophy. Acta, v.637, p.8-17, 1981.

[5] BRITTON, G. UV/Visible Spectroscopy. In: BRITTON, G.; LIAAEN-JENSEN, S.; PFANDER, H. (Eds.) Carotenoids: Spectroscopy. Basel: Birkhäuser, 1995. vol 1B, p. 1362.

[6] BRITTON, G.; YOUNG, A.J. Methods for the Isolation and Analysis of Carotenoids. In: YOUNG, A.; BRITTON, G. (Eds.) Carotenoids in Photosynthesis. London: Chapman \& Hall, 1993. p. 409-457.

[7] BUREAU, J.L.; BUSHWAY, R.J. HPLC determination of carotenoids in fruits and vegetables in the United States. J. Food Sci., v.51, p. 128-30, 1986.

[8] BUSHWAY, R.J. Separation of carotenoids in fruits and vegetables by high performance liquid chromatography. J. Liq. Chromatogr., v. 8, p. 1527-47, 1985.

[9] BUSHWAY, R.J. Determination of $\alpha$ - and $\beta$-carotene in some raw fruits and vegetables by high-performance liquid chromatography. J. Agric. Food Chem., v.34, p. 409-12, 1986.

[10] CARVALHO, P.R.N.; COLLINS, C.H.; RODRIGUEZ-AMAYA, D.B. Comparison of provitamin A determination by normal-phase gravity-flow column chromatography and reversed-phase high performance liquid chromatography. Chromatographia, v.33, p.133-7, 1992.

[11] CHANDLER, L.A.; SCHWARTZ, S.J. HPLC separation of cis - trans carotene isomers in fresh and processed fruits and vegetables. J. Food Sci., v. 52, p. 669-72, 1987.

[12] CHANDLER, L.A.; SCHWARTZ, S.J. Isomerization and losses of trans- $\beta$-carotene in sweet potatoes, as affected by processing treatments. J. Agric. Food Chem., v. 36, p. 129-33, 1988.

[13] DAVIES, B.H. Carotenoids. In: GOODWIN, T.W. (Ed.) Chemistry and Biochemistry of Plant Pigments. London: Academic Press. Vol 2, p. 38-165, 1976.

[14] ENZELL, C. R. Mass spectrometric studies of carotenoids. Pure Appl. Chem. v. 20, p. 497-515, 1969.

[15] ENZELL, C.R.; FRANCIS, G.W.; LIAAEN-JENSEN, S. MasS spectrometric studies of carotenoids. 2. Survey of fragmentation reactions. Acta Chem. Scand. v. 3, p. 72750,1969

[16] ENZELL, C.R.; BACK, S. Mass Spectrometry. In: BRITTON, G.; LIAAEN-JENSEN, S.; PFANDER, H. (Eds.) Carotenoids: Spectroscopy. Basel: Birkhäuser, 1995. vol 1B, p. 261-320.

[17] GODOY, H.T.; RODRIGUEZ-AMAYA, D.B. Occurrence of cisisomers of provitamin A in Brazilian fruits. J. Agric. Food Chem., v.42, p.1306-13, 1994.

[18] GOODWIN, T.W. Distribution of Carotenoids. In GOODWIN, T.W. (Ed.) Chemistry and Biochemistry of Plant Pigments. 2nd ed. London: Academic Press, 1976. p 225-57.
[19] GRANADO, F.; OLMEDILLA, B.; BLANCO, I.; ROJASHIDALGO, E. Carotenoid composition in raw and cooked Spanish vegetables. J. Agric. Food Chem., v.40, p. 213540, 1992.

[20] GROSS, J. Pigments in Fruits. London, Academic Press, 1987. p. 87-258.

[21] HEINONEN, M.I.; OLLIAINEN, V.; LINKOLA, E.K.; VARO, P.T.; KOIVISTOINEN, P.E. Carotenoids in Finish foods: vegetables, fruits, and berries. J. Agric. Food Chem., v. 37, p. 655-9, 1989.

[22] KHACHIK, F.; BEECHER, G.R.; WHITTAKER, N.F. Separation, identification and quantification of the major carotenoid and chlorophyll constituents in extracts of several green vegetables by liquid chromatography. J. Agric. Food Chem., v.34, p. 603-16, 1986.

[23] LIAAEN-JENSEN, S. Combined Approach: Identification and Structure Elucidation of Carotenoids. In: BRITTON, G.; LIAAEN-JENSEN, S.; PFANDER, H. (Eds.) Carotenoids: Spectroscopy. Basel: Birkhäuser, 1995. vol 1B, p. 343354.

[24] MERCADANTE, A.Z.; RODRIGUEZ-AMAYA, D.B. Carotenoid composition of a leafy vegetable in relation to some agricultural variables. J. Agric. Food Chem., v.39, p.10947, 1991.

[25] MERCADANTE, A.Z.; RODRIGUEZ-AMAYA, D.B. Carotenoid composition and vitamin A value of some native Brazilian green leafy vegetables. Intern. J. Food Sci. and Technol., v.25, p.213-9, 1990.

[26] MERCADANTE, A.Z.; RODRIGUEZ-AMAYA, D.B.; BRITTON, G. HPLC and mass spectrometric analysis of carotenoids from mango. J. Agric. Food Chem., v.45, p.120-3, 1997.

[27] MERCADANTE, A.Z.; BRITTON, G.; RODRIGUEZ-AMAYA, D.B. Carotenoids from yellow passion fruit (Passiflora edulis). J. Agric. Food Chem., v.46, p.4102-6, 1998.

[28] MERCADANTE, A.Z.; STECK, A.; PFANDER, H. Carotenoids from guava (Psidium guajava L.): isolation and structure elucidation. J. Agric. Food Chem., v. 47, p. 145-151, 1999.

[29] MERCADANTE, A.Z.; STECK, A.; RODRIGUEZ-AMAYA, D.B; PFANDER, H.; BRITTON, G. Isolation of methyl 9'Z-apo6'-lycopenaote from Bixa orellana. Phytochem., v. 41, p. 1201-1203, 1996.

[30] MERCADANTE, A.Z.; STECK, A.; PFANDER, H. Isolation and identification of new apocarotenoids from annatto (Bixa orellana L.) seeds. J. Agric. Food Chem., v. 45, p. 10501054, 1997.

[31] MERCADANTE, A.Z.; STECK, A.; PFANDER, H. Isolation and structure elucidation of minor carotenoids from annatto (Bixa orellana L.) seeds. Phytochem., v. 46, p. 1379-1383, 1997.

[32] MERCADANTE, A.Z.; STECK, A.; PFANDER, H. Three minor carotenoids from annatto (Bixa orellana L.) seeds. Phytochem., v. 52, p. 135-139, 1999.

[33] NYAMBAKA, H.; RYLEY, J. An isocratic reversed-phase HPLC separation of the stereoisomers of the provitamin $A$ carotenoids ( $\alpha$ - and $\beta$-carotene) in dark green vegetables. Food Chem., v.55, p.63-72, 1996.

[34] RAMOS, D.M.R.; RODRIGUEZ-AMAYA, D.B. Determination of the vitamin A value of common Brazilian leafy vegetables. J. Micronutr. Anal. v.3, p.147-55, 1987.

[35] RODRIGUEZ-AMAYA, D.B.; KIMURA, M.; GODOY, H.T.; ARIMA, H.K. Assessment of provitamin A determination by open column chromatography/visible absorption spectrometry. J. Chromatogr. Sci., v.25, p.624-9, 1988. 
[36] ROUCHAUD, J.; MOONS, C.; MEYER, J.A. The effects of selected herbicide and fungicide treatments on the carotenes and xanthophylls in lettuce. J. Hort. Sci., v.60, p.245-9, 1985.

[37] TEE, E.-S.; LIM, C.-L. Carotenoid composition and content of Malaysian vegetables and fruits by the AOAC and HPLC methods. Food Chem., v. 41, p. 309-39, 1991.

[38] TSUKIDA, K.; SAIKI, K.; TAKII, T.; KOYAMA, Y. Structural elucidation of the main cis- $\beta$-carotenes. J. Nutr. Sci. Vitaminol., v. 27, p. 551-561, 1981.
[39] YAMAUCHI, N.; WATADA, A.E. Pigment changes in parsley leaves during storage in controlled or ethylene containing atmosphere. J. Food Sci., v.58, p.616-8, 1993.

\section{6 - AGRADECIMENTOS}

Os autores agradecem o auxílio financeiro do MCT/ FINEP/PRONEX. 